\title{
NON-AMENABLE PRINCIPAL GROUPOIDS WITH WEAK CONTAINMENT
}

\author{
VADIM ALEKSEEV AND MARTIN FINN-SELL
}

\begin{abstract}
We construct examples of principal groupoids that have weak containment but are not amenable, thus answering questions by Claire AnantharamanDelaroche and Rufus Willett.
\end{abstract}

\section{Amenability and Weak CONTAinment}

Through its many guises, amenability of a group has become a focal concept within both group theory and operator algebras. By a classical result of Andrzej Hulanicki ([Hul64]), the amenability of a discrete group is equivalent to the property that all unitary representations of the group are weakly contained in the left regular representation - we refer to this property as weak containment.

Recently, there has been interest in how far Hulanicki's classical result can be generalised, and in particular it has been shown by Rufus Willett in Wil15 to fail for groupoids that are bundles of groups. The purpose of this note is to address Question 4.1 from [AD16] (that was also raised in Remark 3.6 of [Wil15]), namely we give an example of a principal groupoid that has weak containment, but is not amenable.

For a information about étale groupoids, their $C^{*}$-algebras and representations, we suggest [BO08, Chapter 5]. For more general information concerning locally compact groupoids, we refer to [Ren80] and [ADR00] and references therein.

1.1. Preliminaries. Throughout the text, $\mathcal{G}$ will be an étale Hausdorff topological groupoid, and for any subset of the unit space $U \subset \mathcal{G}^{(0)}$ we will denote by $\left.\mathcal{G}\right|_{U}$ the restriction of $\mathcal{G}$ to $U$, i.e the subgroupoid of $\mathcal{G}$ consisting of all the elements with both source and range in $U$. We remark that this groupoid is open (resp. closed) if $U$ is open (resp. closed) in $\mathcal{G}^{(0)}$.

Definition 1.1. $\mathcal{G}$ has weak containment if the left regular representation $\lambda$ : $C^{*}(\mathcal{G}) \rightarrow C_{r}^{*}(\mathcal{G})$ is a $*$-isomorphism.

From [ADR00, Theorem 6.1.4] it is known that all measurewise amenable groupoids have weak containment. We recall the general strategy used in Wil15 to construct a non-amenable groupoid with weak containment.

Definition 1.2. Let $\Gamma$ be a residually finite finitely generated discrete group and let $\mathcal{N}:=\left\{N_{i}\right\}_{i}$ be a family of nested, finite index normal subgroups of $\Gamma$ with trivial intersection. Let $\pi_{i}$ be the quotient map $\Gamma \rightarrow \Gamma / N_{i}$. The HLS groupoid $\mathcal{G}$ associated to $\Gamma$ and $\mathcal{N}$ is:

$$
\mathcal{G}:=\bigsqcup_{i \in \mathbb{N}^{+}}\{i\} \times X_{i}
$$

2010 Mathematics Subject Classification. 22A22, $46 \mathrm{~L} 55$.

${ }^{1}$ After Nigel Higson, Vincent Lafforgue and George Skandalis who first considered this construction for a related purpose in [HLS02]. 
where

$$
X_{i}=\left\{\begin{array}{l}
\Gamma / N_{i} \text { if } i \in \mathbb{N} \\
\Gamma \text { if } i=\infty
\end{array}\right.
$$

equipped with the topology generated by the following sets:

- the singletons $\{(i, g)\}$;

- the tails: $\left\{\left(i, \pi_{i}(g)\right) \mid i \in \mathbb{N}^{+}, i>N\right\}$ for every fixed $g \in \Gamma$ and $N \in \mathbb{N}$.

One can check that equipped with this topology and the obvious partially defined product and inverse $\mathcal{G}$ becomes an étale, locally compact Hausdorff groupoid with unit space $\mathbb{N}^{+}$. Moreover, it is amenable if and only if $\Gamma$ is amenable.

Considering the open invariant set $U:=\mathbb{N} \subset \mathcal{G}^{(0)}$, we obtain a commuting diagram with exact rows consisting of $C^{*}$-algebras associated with the restriction groupoids $\left.\mathcal{G}\right|_{U}$ and $\left.\mathcal{G}\right|_{U^{c}}$ :

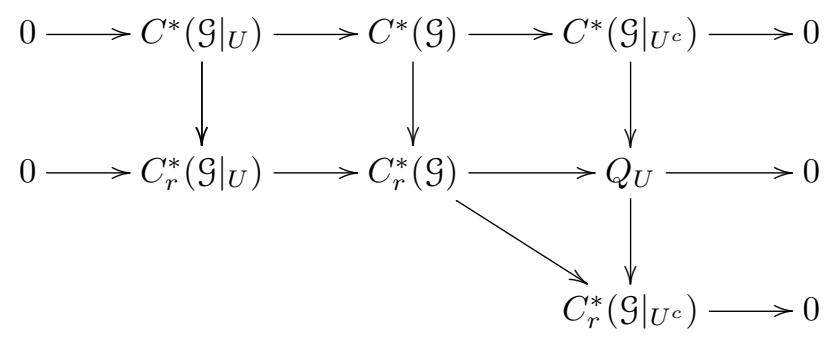

where $Q_{U}$ is the quotient by the ideal $C_{r}^{*}\left(\left.\mathcal{G}\right|_{U}\right)$. The groupoid $\left.\mathcal{G}\right|_{U}$ is amenable and therefore has weak containment, and so to deduce weak containment for $\mathcal{G}$ it is enough to show that the map $C^{*}\left(\left.\mathcal{G}\right|_{U^{c}}\right) \rightarrow Q_{U}$ is isometric. In Wil15, it is then proved that $C^{*}\left(\left.\mathcal{G}\right|_{U^{c}}\right) \cong C^{*}(\Gamma), C_{r}^{*}\left(\left.\mathcal{G}\right|_{U^{c}}\right) \cong C_{r}^{*}(\Gamma)$, and that vertical arrows come from canonical maps between these; therefore weak containment is automatic if $\Gamma$ is amenable. In the non-amenable case, weak containment is deduced from the property FD of Lubotzky-Shalom ([LS04]):

Definition 1.3. Let $\Gamma$ be a countable discrete group. $\Gamma$ has property $F D$ if finite dimensional representations are dense in the unitary dual of $\Gamma$; a family of finite quotients $\mathcal{X}:=\left\{\Gamma / N_{\kappa}\right\}_{\kappa}$ is an FD family if the set of representations of $\Gamma$ which factor through the quotient maps $\left\{\pi_{\kappa}: \Gamma \rightarrow \Gamma / N_{\kappa}\right\}_{\kappa}$ is dense in the unitary dual of $\Gamma$.

This is then used to deduce the key result in Wil15: if $X$ is an FD family, then the $C^{*}$-algebra $Q_{U}$ in the corresponding HLS groupoid $\mathcal{G}$ is isomorphic to the maximal group $C^{*}$-algebra $C^{*}(\Gamma)$ through the vertical map $C^{*}(\Gamma) \cong C^{*}\left(\left.\mathcal{G}\right|_{U^{c}}\right) \rightarrow$ $Q_{U}$ in the above diagram. However, non-amenability of $\Gamma$ implies that the groupoid $\mathcal{G}$ is non-amenable, and this finishes the construction.

\section{Constructing examples}

Let $\Gamma$ be a non-amenable residually finite group with a countable nested (FD) family $\mathcal{X}$ and let $\mathcal{G}$ be the HLS groupoid from the previous section. We are going to consider a transformation groupoid constructed from $\mathcal{G}$ and the set of finite quotients $X$. Let $X:=\bigsqcup_{i} X_{i}$. We begin by constructing the unit space for this groupoid as a second countable compactification of $X$.

For $g \in X_{i}$, consider the shadow of $g$ in $X$ :

$$
\operatorname{Sh}(g):=\bigcup_{j \geqslant i}\left\{x \in X_{j} \mid \pi_{i, j}(x)=g\right\},
$$

where $\pi_{i, j}: \Gamma / N_{j} \rightarrow \Gamma / N_{i}$ is the canonical quotient map. 
Let $B$ be the $\mathcal{G}$-invariant $C^{*}$-subalgebra of $\ell^{\infty}(X)$ generated by $\left\{\delta_{x}\right\}_{x \in X}$ and the sets of projections $\left\{\mathbf{1}_{\mathrm{Sh}(g))}\right\}_{g \in X_{i}}$ for all $i \in \mathbb{N}$. We will consider the spectrum of $B$, which we denote by $\widehat{X}$. As $B$ is $\mathcal{G}$-invariant, $\widehat{X}$ carries a natural $\mathcal{G}$-action, and we consider the transformation groupoid $G:=\widehat{X} \rtimes \mathcal{G}$.

We remark that $G^{(0)}$ contains a obvious open invariant subset $X \subset G^{(0)}$ corresponding to the ideal generated by $\delta_{x}, x \in X$, and let $\partial X \subset G^{(0)}$ be the closed (compact) complement. The following lemma describes it as a $\Gamma$-space.

\section{Lemma 2.1.}

i) The space $\partial X$ is $\Gamma$-equivariantly homeomorphic to $\widehat{\Gamma}_{x}$, the profinite completion of $\Gamma$ with respect to the family $X$.

ii) The algebra $A:=C(\partial X)$ is a direct limit of finite-dimensional $\Gamma-C^{*}$ algebras $A_{i}$, such that the action on $A_{i}$ factors through $\Gamma / N_{i}$.

Proof. The inclusion $\partial X \subset \widehat{X}$ gives rise to a restriction homomorphism $r: B=$ $C(\widehat{X}) \rightarrow C(\partial X)=A$ which obviously contains all elements $\delta_{x}, x \in X$, in its kernel. Thus, $A=C(\partial X)$ is generated by images of the projections $p_{i, g}:=\mathbf{1}_{\operatorname{Sh}(g)}$, $i \in \mathbb{N}, g \in X_{i}$.

Consider the finite-dimensional $C^{*}$-algebras $A_{i}$ generated by the projections $p_{i, g}$, $g \in X_{i}$. Notice that the action of $\Gamma$ on $A_{i}$ obviously factors through $\Gamma / N_{i}$, as it is isomorphic to the natural left action of $\Gamma$ on $\mathbb{C}\left[\Gamma / N_{i}\right]$. Moreover, there are natural $\Gamma$-equivariant injective homomorphisms

$$
\begin{gathered}
\rho_{i, j}: A_{i} \rightarrow A_{j}, \\
\rho_{i, j}\left(p_{i, g}\right)=\sum_{\pi_{i, j}\left(g^{\prime}\right)=g} p_{j, g^{\prime}}
\end{gathered}
$$

corresponding to ( $\Gamma$-equivariant) projections $\Gamma / N_{j} \rightarrow \Gamma / N_{i}$.

Furthermore, the element $p_{i, g}-\rho_{i, i+1}\left(p_{i, g}\right)$, considered as an element of $C(\widehat{X})$, equals $\delta_{g}$, and therefore the kernel of the restriction map $r$ is generated by such differences. As a consequence, we get a $\Gamma$-equivariant isomorphism $A \cong \lim A_{i}$, whence the boundary $\partial X$ is the inverse limit of the corresponding projective system of $\Gamma$-spaces. By the remark above, this projective system of spaces is naturally identified with the projective system $\left\{\Gamma / N_{i}\right\}_{i \in \mathbb{N}}$, equipped with the natural left $\Gamma$-action. This finishes the proof.

Remark 2.2. As the $C^{*}$-algebras $A_{i}$ are finite-dimensional, they have natural regular representations $\lambda_{i}: A_{i} \rightarrow \mathbb{B}\left(A_{i}\right)$, where $A_{i}$ carries the Hilbert space structure obtained from the natural trace $\tau_{i}: p_{i, g} \mapsto 1$ as well as a unitary representation $\alpha_{i}: \Gamma \rightarrow \mathcal{U}\left(A_{i}\right)$ given by the $\Gamma$-action. Let $\phi_{i}$ be the bijection that sends $p_{i, g}$ to $\pi_{i}(g)$. This induces an isomorphism $\phi_{i}$ between $A_{i} \rtimes \Gamma / N_{i}$ and the full matrix algebra $\mathbb{M}_{\left|X_{i}\right|}$.

The consequence of Lemma 2.1 is that we can identify the boundary piece of $G$ as

$$
\left.G\right|_{\partial X} \cong \widehat{\Gamma}_{x} \rtimes \Gamma
$$

where the latter groupoid is the transformation groupoid with the natural free action. It follows that $G$ is a principal groupoid as the action on $X$ is obviously free: $\left.G\right|_{X} \cong \bigsqcup_{i \in \mathbb{N}}\left(X_{i} \rtimes \Gamma / N_{i}\right)$ by construction.

Attached with this decomposition of $\widehat{X}$ into $X$ and $\partial X$ we obtain a commuting diagram with exact rows: 


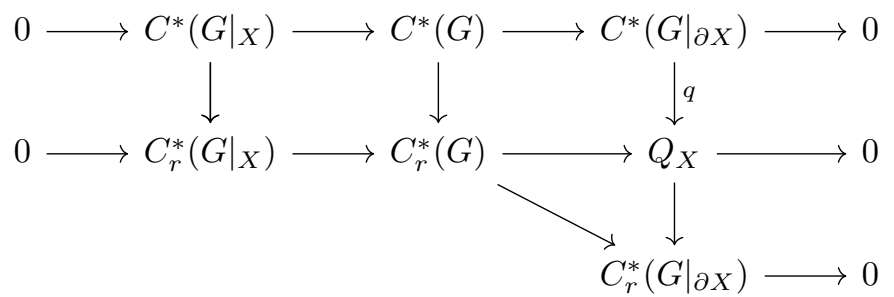

\section{Lemma 2.3.}

i) If the map $q: C^{*}\left(\left.G\right|_{\partial X}\right) \rightarrow Q_{X}$ in the above diagram is an isomorphism, then $G$ has weak containment;

ii) If the $\operatorname{map} Q_{X} \rightarrow C_{r}^{*}\left(\left.G\right|_{\partial X}\right)$ in the above diagram is not an isomorphism, then $G$ is non-amenable.

Proof. As $\left.G\right|_{X} \cong \bigsqcup_{i} X_{i} \rtimes \Gamma / N_{i}$ is a disjoint union of pair groupoids with the obvious discrete topology, it is amenable and therefore has weak containment. i) now follows from the above diagram by the five lemma. ii) follows as amenability passes to restrictions to closed invariant subsets.

As a final preliminary before proving our result, we describe an ambient setting for $Q_{X}$ and $C_{r}^{*}(G)$.

Lemma 2.4. There is a natural isometric embedding

$$
C_{r}^{*}(G) \hookrightarrow \prod_{i \in \mathbb{N}} \mathbb{M}_{\left|X_{i}\right|}
$$

which induces an isometric embedding

$$
\iota: Q_{X} \hookrightarrow \frac{\prod_{j} \mathbb{M}_{\left|X_{j}\right|}}{\bigoplus_{j} \mathbb{M}_{\left|X_{j}\right|}}
$$

Proof. Since $X$ is dense in $\widehat{X}$, we can use [KS04, Corollary 2.4 a)] to see that the norm of an element $f \in C_{r}^{*}(G)$ is equal to $\sup _{x \in X}\left\|\lambda_{x}(f)\right\|$, where $\lambda_{x}$ is the left regular representation on $s^{-1}(x)$ (which is equal to $X_{i}$ if $x \in X_{i}$ ). Thus we get a natural embedding

$$
C_{r}^{*}(G) \hookrightarrow \prod_{i \in \mathbb{N}} \mathbb{M}_{\left|X_{i}\right|}
$$

where $\mathbb{M}_{\left|X_{i}\right|}$ is the full matrix algebra over $X_{i}$ (viewed as bounded operators on $\left.\ell^{2}\left(X_{i}\right)\right)$. As $\left.G\right|_{X}$ is a union of pair groupoids, we get $C_{r}^{*}\left(\left.G\right|_{X}\right) \cong \bigoplus_{j} \mathbb{M}_{\mid X_{j}}$, which implies that $Q_{X}$ is isometrically embedded into $\frac{\prod_{j} \mathbb{M}_{\left|X_{j}\right|}}{\bigoplus_{j} \mathbb{M}_{\left|X_{j}\right|}}$.

Our goal now is connect the maximal crossed product of $A$ by $\Gamma$ with $Q_{X}$ using that $\Gamma$ has property $(\mathrm{FD})$.

Proposition 2.5. The maximal crossed product $A_{i} \rtimes \Gamma$ embeds into $\prod_{j \geqslant i} A_{j} \rtimes \Gamma / N_{j}$ by the natural maps $\rho_{i, j} \rtimes \pi_{j}$.

Proof. The claim is equivalent to the statement that every representation of $A_{i} \rtimes \Gamma$ is weakly contained in a representation factoring through $A_{j} \rtimes \Gamma / N_{j}$. To this end, consider an arbitrary representation $\sigma: A_{i} \rtimes \Gamma \rightarrow \mathbb{B}(\mathcal{H})$ and an element

$$
x=\sum_{g \in \Gamma / N_{i}} p_{i, g} f_{g} \in A_{i} \rtimes_{\text {alg }} \Gamma,
$$


where $f_{g} \in \mathbb{C}[\Gamma]$ and let $\xi, \eta \in \mathcal{H}$ be arbitrary vectors. We have

$$
\langle x \xi, \eta\rangle=\sum_{g \in \Gamma / N_{i}}\left\langle f_{g} \xi, p_{i, g} \eta\right\rangle
$$

By property (FD) of $\Gamma$ for every $\varepsilon>0$ we get a $j \geqslant i$, representation $\sigma^{\prime}: \Gamma \rightarrow$ $\Gamma / N_{j} \rightarrow \mathcal{U}\left(\mathcal{H}^{\prime}\right)$ and vectors $\xi_{1}^{\prime}, \ldots, \xi_{N}^{\prime}, \eta_{1}^{\prime}, \ldots, \eta_{N}^{\prime} \in \mathcal{H}^{\prime}$ such that

$$
\left|\langle x \xi, \eta\rangle-\sum_{g \in \Gamma / N_{i}} \sum_{\ell=1}^{N}\left\langle\sigma^{\prime}\left(f_{g}\right) \xi_{\ell}^{\prime}, \eta_{\ell}^{\prime}\right\rangle\right|<\varepsilon .
$$

Consider now the Hilbert space $\mathcal{H}^{\prime \prime}:=\mathcal{H}^{\prime} \otimes A_{j}$ and the representation $\sigma^{\prime \prime}:=$ $\sigma^{\prime} \otimes \alpha_{j}: \Gamma \rightarrow \mathcal{U}\left(\mathcal{H}^{\prime \prime}\right)$ (which factors through $\Gamma / N_{j}$ ) as well as the representation $m_{i, j}:=\operatorname{id}_{\mathcal{H}^{\prime}} \otimes\left(\lambda_{j} \circ \rho_{i, j}\right): B_{i} \rightarrow \mathbb{B}\left(\mathcal{H}^{\prime \prime}\right)$. It's easy to see that these give a covariant pair and that for every $h \in \Gamma / N_{j}$ we have an equality of matrix coefficients

$$
\left\langle\sigma^{\prime}\left(f_{g}\right) \xi_{\ell}^{\prime}, \eta_{\ell}^{\prime}\right\rangle=\left\langle\sigma^{\prime \prime}\left(f_{g}\right)\left(\xi_{\ell}^{\prime} \otimes p_{j, h}\right), \eta_{\ell}^{\prime} \otimes \sum_{g^{\prime} \in \Gamma / N_{j}} p_{j, g^{\prime}}\right\rangle
$$

Therefore any matrix coefficient of any representation of $A_{i} \rtimes \Gamma$ is approximated by a matrix coefficient of a representation factoring through $A_{j} \rtimes \Gamma / N_{j}$ for a suitable $j$, which ends the proof.

We now can prove the following:

Proposition 2.6. The maximal crossed product $A \rtimes \Gamma$ is isomorphic to $Q_{X}$ through the canonical quotient map $q: A \rtimes \Gamma \rightarrow Q_{X}$.

Proof. In view of Lemma 2.4, it is enough to prove that the composition

$$
\iota \circ q: A \rtimes \Gamma \rightarrow \frac{\prod_{j} \mathbb{M}_{\left|X_{j}\right|}}{\bigoplus_{j} \mathbb{M}_{\left|X_{j}\right|}}
$$

is isometric. By Lemma 2.1 and the continuity of the maximal crossed product functor, for this it is enough to prove that the map $\iota \circ q$ is isometric on $A_{i} \rtimes \Gamma$.

To this end, take an arbitrary element of the algebraic crossed product $A_{i} \rtimes_{\text {alg }} \Gamma$

$$
z=\sum_{g \in \Gamma / N_{i}} p_{i, g} f_{g}
$$

where $f_{g} \in \mathbb{C}[\Gamma]$, and observe that it lifts to $C^{*}(G)$ as the family of elements

$$
\left(z_{j}\right)_{j}=\left(\left.\sum_{g \in \Gamma / N_{i}} p_{i, g}\right|_{X_{j}} \pi_{j}\left(f_{g}\right)\right)_{j} \in C\left(X_{j}\right) \rtimes \Gamma / N_{j}, \quad j \geqslant i .
$$

Using the isomorphisms $\phi_{j}: A_{j} \rtimes \Gamma / N_{j} \rightarrow \mathbb{M}_{\left|X_{j}\right|}$ defined in Remark 2.2, we now see that the image of $z$ under the composition $\iota \circ q$ coincides with $\left(\phi_{j} \circ\left(\rho_{i, j} \rtimes \pi_{j}\right)\right)(z) \in$ $\prod_{j \geqslant i} \mathbb{M}_{\left|X_{j}\right|}$, because $\rho_{i, j}\left(p_{i, g}\right)(x)=p_{i, g}(x)$ for all $x \in X_{j}$.

Therefore the map $\iota \circ q: A \rtimes \Gamma \rightarrow \frac{\prod_{j} \mathbb{M}_{\left|X_{j}\right|}}{\bigoplus_{j} \mathbb{M}_{\left|X_{j}\right|}}$ coincides with the map $A \rtimes \Gamma \rightarrow$ $\frac{\prod_{j} \mathbb{M}_{\left|X_{j}\right|}}{\bigoplus_{j} \mathbb{M}_{\left|X_{j}\right|}}$ induced by $\phi_{j} \circ\left(\rho_{i, j} \rtimes \pi_{j}\right)$. The latter is isometric by the previous proposition, and therefore we are done.

Theorem 2.7. Let $\Gamma$ be a non-amenable residually finite group with a countable nested (FD) family $X$. Then the groupoid $G$ constructed above is principal and non-amenable, but has weak containment. 
VADIM ALEKSEEV AND MARTIN FINN-SELL

Proof. In view of Lemma 2.3 and Proposition 2.6, it remains to prove that the map $Q_{X} \rightarrow C_{r}^{*}\left(\left.G\right|_{\partial X}\right)$ is not an isomorphism. We remark that $\partial X$ has a $\Gamma$-invariant probability measure obtained by taking the weak* limit of the normalised counting measures on each $X_{i}$. By [WY14, Lemma 7.1, Remark 7.1], we get that $Q_{X}$ contains $C^{*}(\Gamma)$ as a *-subalgebra, which maps onto $C_{r}^{*}(\Gamma)$ under the quotient map $Q_{X} \rightarrow$ $C_{r}^{*}\left(\left.G\right|_{\partial X}\right)$. Hence the map $Q_{X} \rightarrow C_{r}^{*}\left(\left.G\right|_{\partial X}\right)$ is not an isomorphism. This finishes the proof.

We remark that [LS04, Theorems 2.2 and 2.8] give a wealth of examples of $\Gamma$ that satisfy the conditions above: notably free groups and surface groups (also cyclic extensions of these groups).

\section{ACKNOWLEDGEMENTS}

This question was asked to the authors by Rufus Willett at the Erwin Schrödinger Institute programme on "Measured group theory", February 2016. Correspondingly, the authors would like to thank both Rufus Willett for sharing the question with us and the ESI for its support. This work was also partially supported by the ERC grant "ANALYTIC" no. 259527 of Goulnara Arzhantseva.

\section{REFERENCES}

[AD16] Claire Anantharaman-Delaroche. Some remarks about the weak containment property for groupoids and semigroups. arXiv:1604.01724 [math], April 2016.

[ADR00] C. Anantharaman-Delaroche and J. Renault. Amenable groupoids, volume 36 of Monographies de L'Enseignement Mathématique [Monographs of L'Enseignement Mathématique]. L'Enseignement Mathématique, Geneva, 2000. With a foreword by Georges Skandalis and Appendix B by E. Germain.

[BO08] Nathanial P. Brown and Narutaka Ozawa. $C^{*}$-algebras and finite-dimensional approximations, volume 88 of Graduate Studies in Mathematics. American Mathematical Society, Providence, RI, 2008.

[HLS02] N. Higson, V. Lafforgue, and G. Skandalis. Counterexamples to the Baum-Connes conjecture. Geometric \& Functional Analysis GAFA, 12(2):330-354, June 2002.

[Hul64] A. Hulanicki. Groups whose regular representation weakly contains all unitary representations. Studia Math., 24:37-59, 1964.

[KS04] Mahmood Khoshkam and Georges Skandalis. Crossed products of $C^{*}$-algebras by groupoids and inverse semigroups. Journal of Operator Theory, 51(2):255-279, 2004.

[LS04] Alexander Lubotzky and Yehuda Shalom. Finite representations in the unitary dual and Ramanujan groups. In Discrete geometric analysis, volume 347 of Contemp. Math., pages 173-189. Amer. Math. Soc., Providence, RI, 2004.

[Ren80] Jean Renault. A groupoid approach to $C^{*}$-algebras, volume 793 of Lecture Notes in Mathematics. Springer, Berlin, 1980.

[Wil15] Rufus Willett. A non-amenable groupoid whose maximal and reduced $C^{*}$-algebras are the same. arXiv:1504.05615 [math], April 2015.

[WY14] Rufus Willett and Guoliang Yu. Geometric property (T). Chinese Annals of Mathematics. Series B, 35(5):761-800, 2014.

Vadim Alekseev, Technische Universität Dresden, Fachrichtung Mathematik, Institut für Geometrie, 01062, Dresden, Deutschland

E-mail address: vadim.alekseev@tu-dresden.de

Martin Finn-Sell, Universität Wien, Fakultät für Mathematik, Oskar-MorgensternPlatz 1, 1090 Wien, Österreich

E-mail address: martin.finn-sell@univie.ac.at 